\title{
Cap-Cosets and Cup-Cosets of a Subset $S$ in an Artex Space A over A Bi-Monoid M
}

\author{
K. Muthukumaran ${ }^{1}$, M. Kamaraj ${ }^{2}$ \\ ${ }^{1}$ Assistant Professor / P.G. and Research Department of Mathematics/Saraswathi Narayanan College, \\ Perungudi Madurai-625022, Tamil Nadu, India. \\ ${ }^{2}$ Associate Professor / Department Of Mathematics / Government Arts College, Melur, Madurai-625106, \\ Tamil Nadu, India.
}

\begin{abstract}
We define a Cap-coset $a^{\wedge} S$ of a subset $S$ in an Artex space $A$ over a Bi-monoid $M$ and a Cup-coset a $v S$ of a subset $S$ in an Artex space A over a Bi-monoid $M$. We prove $a^{\wedge} S$ need not be equal to $S$ even when $S$ is a SubArtex Space of $A$ and $a \epsilon S$. We prove if a $\epsilon S$, then $a^{\wedge} S \underline{C} S$. We prove that for $a, b \epsilon S$ a $\leq b$ implies $a^{\wedge} S$ $\underline{C} b^{\wedge} S$. We also prove that $a v S$ need not be equal to $S$ even when $S$ is a SubArtex Space of $A$ and $a \epsilon S$. We prove if a $\epsilon S$, then a $\vee S \underline{C} S$. We prove that for $a, b \in S a \leq b$ implies $b \vee S \underline{C}$ a $v S$.
\end{abstract}

Keywords: Artex spaces, Cap-cosets, Cup-cosets, SubArtex Spaces.

\section{Introduction}

The study of left cosets and right cosets in group theory motivated us to bring this paper. The theory of Groups is one of the richest branches of abstract algebra. Groups of transformations play an important role in geometry. A more general concept than that of a group is that of a semi-group. The aim of considering semigroups is to provide an introduction to the theory of rings. Two binary operations are defined for a ring. With respect to the first operation written in the order, it is an abelian group and with respect to the second one, it is enough to be a semi-group. We study many examples of rings and we have so many results in rings. However, there are many spaces or sets which are monoids with respect to two or more operations. This motivated us to define a more general concept bi-monoid than that of a ring. So we can have many algebraic systems which are bi-monoids, but not rings. With these concepts in mind we enter into Lattices. In Discrete Mathematics Lattices and Boolean algebra have important applications in the theory and design of computers. There are many other areas such as engineering and science to which Boolean algebra is applied. Boolean Algebra was introduced by George Boole in 1854. A more general algebraic system is the lattice. A Boolean Algebra is introduced as a special lattice.

The study of Lattices and Boolean algebra together with our bi-monoid motivated us to bring our papers titled "Artex Spaces over Bi-monoids", "Subartex Spaces Of an Artex Space Over a Bi-monoid", "Bounded Artex Spaces Over Bi-monoids and Artex Space Homomorphisms" and "Boolean Artex spaces over bi-monoids". As an another development of it now, we introduce "Cap-cosets and Cup-cosets of a subset $\mathrm{S}$ in an Artex Space A over a Bi-monoid M".

\subsubsection{Definitions}

\section{Preliminaries}

2.1.1 Lattice : A lattice is a partially ordered set ( $\mathrm{L}, \leq$ ) in which every pair of elements $a, b \in \mathrm{L}$ has a greatest lower bound and a least upper bound.

The greatest lower bound of $a$ and $b$ is denoted by $a^{\wedge} b$ and the least upper bound of $a$ and $b$ is denoted by avb

2.1.2 Lattice as an Algebraic System : A lattice is an algebraic system $(\mathrm{L}, \wedge$, v) with two binary operations ^ and $\mathrm{v}$ on $\mathrm{L}$ which are both commutative, associative, and satisfy the absorption laws namely $\mathrm{a}^{\wedge}(\mathrm{a} \vee \mathrm{b})=\mathrm{a}$ and $\quad \mathrm{av}\left(\mathrm{a}^{\wedge} \mathrm{b}\right)=\mathrm{a}$

The operations $\wedge$ and $\mathrm{v}$ are called cap and cup respectively, or sometimes meet and join respectively.

Proposition 2.1.3 : Let $(\mathrm{L}, \leq)$ be a lattice in which ${ }^{\wedge}$ and $\mathrm{v}$ denote the operations of cap and cup respectively. For any a,beL

$$
\mathrm{a} \leq \mathrm{b} \Leftrightarrow \mathrm{a}^{\wedge} \mathrm{b}=\mathrm{a} \Leftrightarrow \mathrm{a} \vee \mathrm{b}=\mathrm{b}
$$

Proposition 2.1.4: Let $(\mathrm{L}, \leq)$ be a lattice in which ${ }^{\wedge}$ and $\mathrm{v}$ denote the operations of cap and cup respectively. For any a,b,c $\epsilon \mathrm{L}$ the following properties called isotonicity hold :

$$
\begin{aligned}
& \mathrm{b} \leq \mathrm{c} \Rightarrow 1 . \mathrm{a}^{\wedge} \mathrm{b} \leq \mathrm{a}^{\wedge} \mathrm{c} \\
& \text { 2. } a v b \leq a v c
\end{aligned}
$$

2.1.5 Bi-monoid : A system $(\mathrm{M},+,$.$) is called a Bi-monoid if$

$$
\text { 1. }(\mathrm{M},+) \text { is a monoid }
$$


2. ( $\mathrm{M},$.$) is a monoid and$

3. a. $(b+c)=a \cdot b+a \cdot c$ and $(a+b) \cdot c=a \cdot c+b \cdot c$, for all $a, b, c \in M$

\subsubsection{Examples}

2.2.1 Let $\mathrm{W}=\{0,1,2,3, \ldots\}$.

Then $(\mathrm{W},+,$.$) , where +$ and. are the usual addition and multiplication respectively, is a bi-monoid.

Here 0 and 1 are the identity elements of $\mathrm{W}$ with respect to addition and multiplication respectively.

2.2.2 Let $\mathrm{S}$ be any set. Consider $\mathrm{P}(\mathrm{S})$, the power set of $\mathrm{S}$.

Then $(\mathrm{P}(\mathrm{S}), \mathrm{U}, \cap)$ is a bi-monoid.

Here $\varphi$ and $S$ are the identity elements of $P(S)$ with respect to $U$ and $\cap$ respectively.

2.2.3 Let $\mathrm{Q}^{\prime}=\mathrm{Q}^{+} \mathrm{U}\{0\}$, where $\mathrm{Q}^{+}$is the set of all positive rational numbers.

Then $\left(\mathrm{Q}^{\prime},+,.\right)$ is a bi-monoid.

2.2.4 Let $\mathrm{R}^{\prime}=\mathrm{R}^{+} \mathrm{U}\{0\}$, where $\mathrm{R}^{+}$is the set of all positive real numbers.

Then $\left(\mathrm{R}^{\prime},+\right.$, . $)$ is a bi-monoid.

\subsubsection{Definition}

2.3.1 Artex Space Over a Bi-monoid : A non-empty set A is said to be an Artex Space Over a bi-monoid $(\mathrm{M},+,$.$) if 1 .(\mathrm{A}, \wedge, \mathrm{v})$ is a lattice and

2.for each $\mathrm{m} \epsilon \mathrm{M}, \mathrm{m} \neq 0$, and $\mathrm{a} \in \mathrm{A}$, there exists an element $\mathrm{ma} \epsilon \mathrm{A}$ satisfying the following

conditions :

(i) $\mathrm{m}\left(\mathrm{a}^{\wedge} \mathrm{b}\right)=m \mathrm{a}^{\wedge} \mathrm{mb}$

(ii) $\mathrm{m}(\mathrm{a} \vee \mathrm{b})=\mathrm{ma} \vee \mathrm{mb}$

(iii) $\mathrm{ma}^{\wedge} \mathrm{na} \leq(\mathrm{m}+\mathrm{n}) \mathrm{a}$ and $\mathrm{ma} v \mathrm{na} \leq(\mathrm{m}+\mathrm{n}) \mathrm{a}$

(iv) (mn) $\mathrm{a}=\mathrm{m}(\mathrm{na})$, for all $\mathrm{m}, \mathrm{n} \in \mathrm{M}, \mathrm{m} \neq 0, \mathrm{n} \neq 0$, and $\mathrm{a}, \mathrm{b} \in \mathrm{A}$

(v) $1 . \mathrm{a}=\mathrm{a}$, for all $\mathrm{a} \in \mathrm{A}$

Here, $\leq$ is the partial order relation corresponding to the lattice $(A, \wedge, v)$

The multiplication ma is called a bi-monoid multiplication with an artex element or simply bi-monoid multiplication in A.

Unless otherwise stated A remains as an Artex space with the partial ordering $\leq$ need not be "less than or equal to" and $\mathrm{M}$ as a bi-monoid with the binary operations + and . need not be the usual addition and usual multiplication.

\subsubsection{Examples}

2.4.1 Let $\mathrm{W}=\{0,1,2,3, \ldots\}$ and $\mathrm{Z}$ be the set of all integers.

Then $(\mathrm{W},+$, . $)$ is a bi-monoid, where + and . are the usual addition and multiplication respectively.

$(\mathrm{Z}, \leq)$ is a lattice in which ${ }^{\wedge}$ and $\mathrm{v}$ are defined by $\mathrm{a}^{\wedge} \mathrm{b}=\operatorname{mini}\{\mathrm{a}, \mathrm{b}\}$ and $\mathrm{a} v \mathrm{~b}=\operatorname{maxi}\{\mathrm{a}, \mathrm{b}\}$, for all $\mathrm{a}, \mathrm{b} \in \mathrm{Z}$.

Clearly for each $\mathrm{m} \in \mathrm{W}, \mathrm{m} \neq 0$, and for each $\mathrm{a} \in \mathrm{Z}$, there exists $\mathrm{ma} \in \mathrm{Z}$.

Also,

(i) $\mathrm{m}\left(\mathrm{a}^{\wedge} \mathrm{b}\right)=\mathrm{ma}^{\wedge} \mathrm{mb}$

(ii) $\mathrm{m}(\mathrm{a} \vee \mathrm{b})=\mathrm{ma} \vee \mathrm{mb}$

(iii) $\mathrm{ma}^{\wedge} \mathrm{na} \leq(\mathrm{m}+\mathrm{n}) \mathrm{a}$ and $\mathrm{ma} v \mathrm{na} \leq(\mathrm{m}+\mathrm{n}) \mathrm{a}$

(iv) $(\mathrm{mn}) \mathrm{a}=\mathrm{m}(\mathrm{na})$, for all $\mathrm{m}, \mathrm{n} \in \mathrm{W}, \mathrm{m} \neq 0, \mathrm{n} \neq 0$ and $\mathrm{a}, \mathrm{b} \in \mathrm{Z}$

(v) $1 . \mathrm{a}=\mathrm{a}$, for all $\mathrm{a} \in \mathrm{Z}$

Therefore, $\mathrm{Z}$ is an Artex Space Over the bi-monoid $(\mathrm{W},+$, . )

2.4.2 As defined in Example 2.4.1, Q, the set of all rational numbers is an Artex space over W

2.4.3 As defined in Example 2.4.1, $\mathrm{R}$, the set of all real numbers is an Artex space over $\mathrm{W}$

2.4.4 Let $\mathrm{Q}^{\prime}=\mathrm{Q}^{+} \mathrm{U}\{0\}$, where $\mathrm{Q}^{+}$is the set of all positive rational numbers.

Then $\left(\mathrm{Q}^{\prime},+\right.$, . $)$ is a bi-monoid.

Now as defined in Example 2.4.1, Q, the set of all rational numbers is an Artex space over Q'

2.4.5 Let $\mathrm{R}^{\prime}=\mathrm{R}^{+} \mathrm{U}\{0\}$, where $\mathrm{R}^{+}$is the set of all positive real numbers.

Then $\left(\mathrm{R}^{\prime},+\right.$, . $)$ is a bi-monoid.

As defined in Example 2.4.1, R, the set of all real numbers is an Artex space over R'

2.4.6 As defined in Example 2.4.1, R, the set of all real numbers is an Artex space over Q'

2.4.7 Let $A$ be the set of all sequences $\left(x_{n}\right)$ in $Z$, where $Z$ is the set of all integers and let $W=\{0,1,2,3, \ldots\}$.

Define $\leq$, an order relation, on A by for $\left(\mathrm{x}_{\mathrm{n}}\right),\left(\mathrm{y}_{\mathrm{n}}\right)$ in $\mathrm{A},\left(\mathrm{x}_{\mathrm{n}}\right) \leq^{\prime}\left(\mathrm{y}_{\mathrm{n}}\right)$ means $\mathrm{x}_{\mathrm{n}} \leq \mathrm{y}_{\mathrm{n}}$, for each $\mathrm{n}$,

where $\leq$ is the usual relation "less than or equal to "

Clearly $\leq$ ' is a partial order relation on A

Now the cap ,cup operations are defined by the following :

$\left(\mathrm{x}_{\mathrm{n}}\right)^{\wedge}\left(\mathrm{y}_{\mathrm{n}}\right)=\left(\mathrm{u}_{\mathrm{n}}\right)$, where $\mathrm{u}_{\mathrm{n}}=\operatorname{mini}\left\{\mathrm{x}_{\mathrm{n}}, \mathrm{y}_{\mathrm{n}}\right\}$, for each $\mathrm{n}$.

$\left(\mathrm{x}_{\mathrm{n}}\right) \mathrm{v}\left(\mathrm{y}_{\mathrm{n}}\right)=\left(\mathrm{v}_{\mathrm{n}}\right)$, where $\mathrm{v}_{\mathrm{n}}=\operatorname{maxi}\left\{\mathrm{x}_{\mathrm{n}}, \mathrm{y}_{\mathrm{n}}\right\}$, for each $\mathrm{n}$.

Clearly $\left(\mathrm{A}, \leq^{\prime}\right)$ is a lattice. 
That is, $(\mathrm{A}, \wedge, \mathrm{v})$ is a lattice.

The bi-monoid multiplication in $\mathrm{A}$ is defined by the following :

For each $m \in W, m \neq 0$, and $x \in A$, where $x=\left(x_{n}\right), m x$ is defined by $m x=m\left(x_{n}\right)=\left(m x_{n}\right)$.

Then clearly A is an Artex space over W.

2.4.8 If $\mathrm{B}$ is the set of all sequences $\left(\mathrm{x}_{\mathrm{n}}\right)$ in $\mathrm{Q}$, where $\mathrm{Q}$ is the set of all rational numbers, then as in Example

2.4.7, $\mathrm{B}$ is an Artex space over W.

2.4.9 As defined in 2.4.8, B is an Artex space over Q'

2.4.10 If $\mathrm{D}$ is the set of all sequences $\left(\mathrm{x}_{\mathrm{n}}\right)$ in $\mathrm{R}$, where $\mathrm{R}$ is the set of all real numbers, then as in Example

2.4.7, D is an Artex space over W.

2.4.11 As defined in 2.4.10, D is an Artex space over Q'

2.4.12 As defined in 2.4.10, D is an Artex space over R'.

\subsection{SubArtex Spaces}

2.5.1 Definition : SubArtex Space : Let $(\mathrm{A}, \wedge, \mathrm{v})$ be an Artex space over a bi-monoid $(\mathrm{M},+,$.

Let $S$ be a nonempty subset of $A$. Then $S$ is said to be a SubArtex space of $A$ if $(S, \wedge, v)$ itself is an Artex space over M.

\subsubsection{Examples}

2.6.1 As in Example 2.4.1, $\mathrm{Z}$ is an Artex space over $\mathrm{W}=\{0,1,2,3, \ldots \ldots\}$ and $\mathrm{W}$ is a subset of $\mathrm{Z}$.

Also $\mathrm{W}$ itself is an Artex space over $\mathrm{W}$ under the operations defined in $\mathrm{Z}$

Therefore, $\mathrm{W}$ is a SubArtex space of $\mathrm{Z}$.

2.6.2 As in Example 2.4.2, $\mathrm{Q}$ is an Artex space over $\mathrm{W}=\{0,1,2,3, \ldots .$.$\} and \mathrm{Z}$ is a subset of $\mathrm{Q}$.

Clearly $\mathrm{Z}$ itself is an Artex space over $\mathrm{W}$ and therefore $\mathrm{Z}$ is a SubArtex space of $\mathrm{Q}$.

$\mathrm{W}$ is also a SubArtex space of $\mathrm{Q}$.

2.6.3 As in Example 2.4.3, $\mathrm{R}$ is an Artex space over $\mathrm{W}=\{0,1,2,3, \ldots .$.$\} and \mathrm{Q}$ is a subset of $\mathrm{R}$

Clearly Q itself is an Artex space over W and therefore Q is a SubArtex space of R over W .

$\mathrm{W}$ is also a SubArtex space of $\mathrm{R}$ over $\mathrm{W}$.

2.6.4 As defined in Example 2.4.6, R, the set of all real numbers is an Artex space over Q' and as in Example

2.4.4,

Q, the set of all rational numbers is an Artex space over Q' .

Therefore, Q is a SubArtex space of R over Q'.

2.6.5 In Examples 2.4.7 and 2.4.8, A is a SubArtex space of B over W.

2.6.6 In Examples 2.4.7, 2.4.8 and 2.4.10, A and B are SubArtex spaces of D over W.

2.6.7 In Examples 2.4.9 and 2.4.11, B is a SubArtex space of D over Q'.

Proposition 2.7.1 : Let $(\mathrm{A}, \wedge, \mathrm{v})$ be an Artex space over a bi-monoid $(\mathrm{M},+,$.

Then a nonempty subset $\mathrm{S}$ of $\mathrm{A}$ is a SubArtex space of $\mathrm{A}$ if and only if $\mathrm{S}$ is closed under the operations ${ }^{\wedge}, \mathrm{V}$

and the bi-monoid multiplication in A.

\section{Cap-cosets and Cup-cosets of a subset $\mathrm{S}$ in an Artex space A over a Bi-monoid $M$ 3.1 A Cap-coset of a subset $S$ in an Artex space A over a Bi-monoid $M$}

be an Artex

3.1.1 Definition : A Cap-coset of a subset $\mathrm{S}$ in an Artex space A over a Bi-monoid M: Let $\left(\mathrm{A},{ }^{\wedge}, \mathrm{v}\right)$

space over a bi-monoid $(\mathrm{M},+,$. ) and $\mathrm{S}$ be a subset of $\mathrm{A}$.

Let $a \in$ A. Define $a^{\wedge} S=\left\{a^{\wedge} s / s \in S\right\}$. Then $a^{\wedge} S$ is called a Cap-coset of $S$ in $A$.

Problem 3.1.2 : $\mathrm{a}^{\wedge} \mathrm{S}$ need not be equal to $\mathrm{S}$ even when $\mathrm{S}$ is a SubArtex Space of $\mathrm{A}$ and $\mathrm{a} \in \mathrm{S}$.

Proof: We prove this by giving an example

Let $\mathrm{W}=\{0,1,2,3, \ldots\}$ and $\mathrm{Z}$ be the set of all integers.

Then $(\mathrm{W},+$, . ) is a bi-monoid, where + and . are the usual addition and multiplication respectively.

Clearly $(Z, \leq)$ is a lattice in which ${ }^{\wedge}$ and $v$ are defined by $\mathrm{a}^{\wedge} \mathrm{b}=\operatorname{mini}\{\mathrm{a}, \mathrm{b}\}$ and $\mathrm{a} v \mathrm{~b}=\operatorname{maxi}\{\mathrm{a}, \mathrm{b}\}$, for all

$a, b \in Z$ and $\leq$ is the relation "less than or equal to"

Then, $\mathrm{Z}$ is an Artex Space Over the Bi-monoid $(\mathrm{W},+$, . )

Now, $W$ is a subset of $Z$.

Also $\mathrm{W}$ itself is an Artex space over $\mathrm{W}$ under the operations defined in $\mathrm{Z}$

Therefore, $\mathrm{W}$ is a SubArtex space of $\mathrm{Z}$.

Let $\mathrm{S}=\mathrm{W}$

Let us take $\mathrm{a}=1$

Now 1 is in the SubArtex Space W of the Artex Space Z over the bi-monoid W.

Then $\mathrm{a}^{\wedge} \mathrm{S}=1^{\wedge} \mathrm{W}=\left\{1^{\wedge} \mathrm{W} / \mathrm{W} \in \mathrm{W}\right\}$

$$
\begin{aligned}
& =\left\{1^{\wedge} 0,1^{\wedge} 1,1^{\wedge} 2,1^{\wedge} 3, \ldots \ldots\right\} \\
& =\{\operatorname{mini} .\{1,0\}, \text { mini. }\{1,1\}, \text { mini. }\{1,2\}, \text { mini. }\{1,3\}, \ldots \ldots\}
\end{aligned}
$$


$=\{0,1\}$

$\neq \mathrm{W}$

$1^{\wedge} \mathrm{W} \neq \mathrm{W}$ even when $1 \in \mathrm{W}$ and $\mathrm{W}$ is a SubArtex Space of $\mathrm{Z}$

Hence for a SubArtex Space $S$ of an Artex Space A over a bi-monoid M, and a $\in S$, a ${ }^{\wedge} S$ need not be equal to S.

Remark: $1^{\wedge} \mathrm{W}$ is a subset of $\mathrm{W}$.

Therefore, we have the following Proposition.

Proposition 3.1.3 : Let $\left(\mathrm{A},{ }^{\wedge}, \mathrm{v}\right)$ be an Artex space over a bi-monoid $(\mathrm{M},+, .$.$) and \mathrm{S}$ be a SubArtex space of A. If $a \in S$, then $a^{\wedge} S \underline{C} S$

Proof : Let $(\mathrm{A}, \wedge, \mathrm{v})$ be an Artex space over a bi-monoid $(\mathrm{M},+,$. ) and let $\mathrm{S}$ be a SubArtex space of A.

Suppose a $\epsilon \mathrm{S}$

To show a^ $\mathrm{S} \underline{\mathrm{C}} \mathrm{S}$

Let $x \in a^{\wedge} \mathrm{S}$

Then $\mathrm{x}=\mathrm{a}^{\wedge} \mathrm{s}$, for some $\mathrm{s} \in \mathrm{S}$

Since $a \in S$ and $s \in S$ and $S$ is a SubArtex space of $A, a^{\wedge} s \in S$ ( By the Proposition 2.7.1)

That is, $x \in S$

Therefore, $a^{\wedge} \mathrm{S} \underline{\mathrm{C}} \mathrm{S}$.

Proposition 3.1.4 : Let $(\mathrm{A}, \wedge, \mathrm{v})$ be an Artex space over a bi-monoid $(\mathrm{M},+,$.$) and \mathrm{S}$ be a SubArtex space of A. Let $a, b \in S$ such that $a \leq b$, then $a^{\wedge} S \subseteq b^{\wedge} S$

Proof : Let $(\mathrm{A}, \wedge, \mathrm{v})$ be an Artex space over a bi-monoid $(\mathrm{M},+, .$.$) and \mathrm{S}$ be a SubArtex space of $\mathrm{A}$.

Let $a, b \in \mathrm{S}$ such that $\mathrm{a} \leq \mathrm{b}$

To show that $\mathrm{a}^{\wedge} \mathrm{S} \mathrm{C} \mathrm{b}^{\wedge} \mathrm{S}$

Let $\mathrm{x} \in \mathrm{a} \wedge \mathrm{S}$

Then $\mathrm{x}=\mathrm{a}^{\wedge} \mathrm{s}$, for some $\mathrm{s} \in \mathrm{S}$

Now since $\mathrm{a} \leq \mathrm{b}, \mathrm{a}^{\wedge} \mathrm{s} \leq \mathrm{b}^{\wedge} \mathrm{s}$ ( By the Proposition 2.1.4 of Isotonicity )

Now $\mathrm{a}^{\wedge} \mathrm{s} \leq \mathrm{b}^{\wedge} \mathrm{s} \quad \Rightarrow\left(\mathrm{a}^{\wedge} \mathrm{s}\right)^{\wedge}\left(\mathrm{b}^{\wedge} \mathrm{s}\right) \quad=\mathrm{a}^{\wedge} \mathrm{s} \quad($ By the Proposition 2.1.3)

$\Rightarrow \quad\left(a^{\wedge} \mathrm{s}\right)^{\wedge}\left(\mathrm{s}^{\wedge} \mathrm{b}\right)=\mathrm{a}^{\wedge} \mathrm{s}$

$\Rightarrow \quad\left(a^{\wedge}\left(s^{\wedge}\left(s^{\wedge} \mathrm{b}\right)\right)\right)=a^{\wedge} \mathrm{s} \quad\left(\right.$ since ${ }^{\wedge}$ is associative $)$

$\left.\Rightarrow\left(a^{\wedge}\left(\left(s^{\wedge} \mathrm{s}\right)^{\wedge} \mathrm{b}\right)\right)\right)=\mathrm{a}^{\wedge} \mathrm{s}$

$\Rightarrow\left(\mathrm{a}^{\wedge}\left(\mathrm{s}^{\wedge} \mathrm{b}\right)\right) \quad=\mathrm{a}^{\wedge} \mathrm{s} \quad\left(\right.$ since $\mathrm{s}$ is idempotent, $\left.\mathrm{s}^{\wedge} \mathrm{s}=\mathrm{s}\right)$

$\Rightarrow\left(\mathrm{a}^{\wedge}\left(\mathrm{b}^{\wedge} \mathrm{s}\right)\right) \quad=\mathrm{a}^{\wedge} \mathrm{s}$

$\Rightarrow\left(a^{\wedge} \mathrm{b}\right)^{\wedge} \mathrm{s} \quad=\mathrm{a}^{\wedge} \mathrm{s}$

$\Rightarrow\left(b^{\wedge} \mathrm{a}\right)^{\wedge} \mathrm{s} \quad=\mathrm{a}^{\wedge} \mathrm{s} \quad\left(\right.$ since ${ }^{\wedge}$ is commutative $)$

$\Rightarrow b^{\wedge}\left(a^{\wedge} s\right) \quad=a^{\wedge} s$

$\Rightarrow \mathrm{b}^{\wedge} \mathrm{s}^{\prime} \quad \quad=\mathrm{a}^{\wedge} \mathrm{s}$, where $\mathrm{s}^{\prime}=\mathrm{a}^{\wedge} \mathrm{s}$

Since $a \in A$ and $s \in S, a^{\wedge} s \in a^{\wedge} S$

Since a $\epsilon$ S, by Proposition 3.1.3, a^ $\mathrm{S} \underline{\mathrm{C}} \mathrm{S}$

Therefore, $\mathrm{s}^{\prime}=\mathrm{a}^{\wedge} \mathrm{s} \in \mathrm{a}^{\wedge} \mathrm{S} \underline{\mathrm{C}} \mathrm{S}$

That is, s' $\in \mathrm{S}$

Therefore, $b^{\wedge} s^{\prime} \in b^{\wedge} S$

That is, $x=a^{\wedge} s=b^{\wedge} s^{\prime} \in b^{\wedge} S$

That is, $x \in b^{\wedge} S$

Hence, $a^{\wedge} S \underline{C} b^{\wedge} S$.

\subsection{A Cup-coset of a subset $\mathrm{S}$ in an Artex space A over a Bi-monoid $\mathrm{M}$}

3.2.1 Definition : A Cup-coset of a subset $\mathrm{S}$ in an Artex space A over bi-monoid $\mathrm{M}:$ Let $(\mathrm{A}, \wedge$, v) be an Artex space over a bi-monoid $(\mathrm{M},+,$. ) and $\mathrm{S}$ be a subset of $\mathrm{A}$.

Let $a \in A$. Define a $v S=\{a \vee s / s \in S\}$. Then a $v$ S is called a Cup-coset of $S$ in $A$.

Problem 3.2.2 : a v $S$ need not be equal to $S$ even when $S$ is a SubArtex Space of $A$ and a $\in$.

Proof: We prove this by giving an example

Let $\mathrm{W}=\{0,1,2,3, \ldots\}$ and $\mathrm{Z}$ be the set of all integers.

Then $(\mathrm{W},+$, . $)$ is a bi-monoid, where + and . are the usual addition and multiplication respectively.

Clearly $(Z, \leq)$ is a lattice in which ${ }^{\wedge}$ and $v$ are defined by $a^{\wedge} b=\operatorname{mini}\{a, b\}$ and $a v b=\operatorname{maxi}\{a, b\}$, for all $a, b \in Z$ and $\leq$ is the relation "less than or equal to"

Then, $\mathrm{Z}$ is an Artex Space Over the Bi-monoid $(\mathrm{W},+,$. )

Now, $W$ is a subset of $Z$.

Also $\mathrm{W}$ itself is an Artex space over $\mathrm{W}$ under the operations defined in $\mathrm{Z}$

Therefore, $\mathrm{W}$ is a SubArtex space of $\mathrm{Z}$. 
Let $\mathrm{S}=\mathrm{W}$

Let us take $\mathrm{a}=5$

Now 5 is in the SubArtex Space W of the Artex Space Z over the bi-monoid W.

Then a $\mathrm{S}=5 \mathrm{v} W=\{5 \mathrm{v} \mathrm{W} / \mathrm{w} \in \mathrm{W}\}$

$$
\begin{aligned}
& =\{5 \mathrm{v} 0,5 \mathrm{v} 1,5 \mathrm{v} 2,5 \mathrm{v} 3, \ldots \ldots\} \\
& =\{\operatorname{maxi} .\{5,0\}, \operatorname{maxi} .\{5,1\}, \operatorname{maxi} .\{5,2\}, \operatorname{maxi} .\{5,3\} \text {, maxi. }\{5,4\}, \operatorname{maxi} .\{5,5\} \text {, } \\
& =\{5,6,7, \ldots \ldots\} \\
& \text { maxi. }\{5,6\} \text {, maxi. }\{5,7\}, \ldots .\}
\end{aligned}
$$

$5 \mathrm{v} W \neq \mathrm{W}$ even when $5 \in \mathrm{W}$ and $\mathrm{W}$ is a SubArtex Space of $\mathrm{Z}$

Hence for a SubArtex Space $S$ of an Artex Space A over a bi-monoid M, and a $\epsilon$, a v $S$ need not be equal to $\mathrm{S}$.

Remark : $5 \mathrm{v} \mathrm{W}$ is a subset of $\mathrm{W}$.

Therefore, we have the following Proposition .

Proposition 3.2.3 : Let $(\mathrm{A}, \wedge$, v) be an Artex space over a bi-monoid $(\mathrm{M},+, .$.$) and \mathrm{S}$ be a SubArtex space of A. If $a \in S$, then a $v \mathrm{~S} \underline{\mathrm{C}} \mathrm{S}$

Proof : Let $(\mathrm{A}, \wedge$, v) be an Artex space over a bi-monoid $(\mathrm{M},+, .$.$) and \mathrm{S}$ be a SubArtex space of A.

Suppose a $\epsilon \mathrm{S}$

To show a v S $\underline{C} S$

Let $x \in$ a $\vee S$

Then $\mathrm{x}=\mathrm{a} v \mathrm{~s}$, for some $\mathrm{s} \in \mathrm{S}$

Since $a \in S$ and $s \in S$ and $S$ is a SubArtex space of A, a v s $\in S \quad$ ( By the Proposition 2.7.1)

That is, $x \in S$

Therefore, a v $\mathrm{S} \underline{\mathrm{C}} \mathrm{S}$.

Proposition 3.2.4 : Let $(\mathrm{A}, \wedge, \mathrm{v})$ be an Artex space over a bi-monoid $(\mathrm{M},+, .$,$) and \mathrm{S}$ be a SubArtex space of A. Let $a, b \in S$ such that $a \leq b$, then $b \vee S C$ a $v S$

Proof : Let $(\mathrm{A}, \wedge, \mathrm{v})$ be an Artex space over a bi-monoid $(\mathrm{M},+, .$.$) and \mathrm{S}$ be a SubArtex space of A.

Let $a, b \in \mathrm{S}$ such that $\mathrm{a} \leq \mathrm{b}$

To show that $b \vee \mathrm{S} \underline{\mathrm{C}}$ a $v \mathrm{~S}$

\begin{tabular}{|c|c|c|c|}
\hline & $(a \vee s) v(b \vee s)$ & $=\mathrm{b} v \mathrm{~s}$ & (By the Proposition 2.1.3) \\
\hline & $(a \vee s) \vee(s \vee b)$ & $=\mathrm{b} \vee \mathrm{s}$ & \\
\hline$\Rightarrow$ & $(\mathrm{a} \vee(\mathrm{s} \vee(\mathrm{s} \vee \mathrm{b}))$ & )$=b \vee s$ & ( since $\mathrm{v}$ is associative ) \\
\hline ᄃ & $(a \vee((s \vee s) \vee b)))$ & $=b \vee s$ & \\
\hline$\Rightarrow$ & $(a \vee(s \vee b))$ & $=\mathrm{b} v \mathrm{~s}$ & (since $\mathrm{s}$ is idempotent, $\mathrm{s} \mathrm{v} \mathrm{s}=\mathrm{s}$ ) \\
\hline & $(\mathrm{a} v(\mathrm{~b} v \mathrm{~s}))$ & $=\mathrm{b} \vee \mathrm{s}$ & ( since $^{\wedge}$ is commutative ) \\
\hline & a v s' & $=\mathrm{b} \vee \mathrm{s}$ & here $s^{\prime}=b$ v s \\
\hline
\end{tabular}

Let $x \in b \vee S$

Then $\mathrm{x}=\mathrm{b} v \mathrm{~s}$, for some $\mathrm{s} \in \mathrm{S}$

Now since $\mathrm{a} \leq \mathrm{b}, \mathrm{a} \vee \mathrm{s} \leq \mathrm{b} \vee \mathrm{s}$ ( By the Proposition 2.1.4 of Isotonicity)

Now a v $\leq$ b v s

Since $b \in A$ and $s \in S, b \vee s \in b \vee S$

Since $b \in \mathrm{S}$, by Proposition 3.2 .3 , b v S C S

Therefore, $s^{\prime}=b \vee s \in b \vee S \underline{C} S$

That is, $s^{\prime} \in \mathrm{S}$

Therefore, a v s' $\epsilon$ a v $S$

That is, $x=b v s=a v s^{\prime} \in$ a v $S$

That is, $x \in a \vee S$

Hence, $b \vee S \underline{C}$ a v S.

\section{Conclusion}

Lattices and Boolean Algebra and The theory of Groups are two different areas in algebra. The concept of right cosests and left cosets in group theory together with the cap operation and the cup operation has given us our new definition Cap-cosets and Cup-cosets of a subset S in an Artex Space A over a bi-monoid M. The definition and the results proved in this paper are very interesting and will be useful. We hope this paper will motivate the researcher to bring new useful results in the theory of Artex spaces over bi-monoids. 


\section{References}

[1] K.Muthukumaran and M.Kamaraj, “Artex Spaces Over Bi-monoids”, "Research Journal of Pure Algebra”, 2(5),May 2012, Pages $135-140$.

[2] K.Muthukumaran and M.Kamaraj, "SubArtex Spaces Of an Artex Space Over a Bi-monoid", "Mathematical Theory and Modeling”, An USA Journal of “International Institute for Science, Technology and Education”, Vol.2, No.7, 2012, pages 39 - 48.

[3] K.Muthukumaran and M.Kamaraj, "Bounded Artex Spaces Over Bi-monoids and Artex Space Homomorphisms", "Research Journal of Pure Algebra”, 2(7), July, 2012, pages 206 - 216

[4] K.Muthukumaran and M.Kamaraj, "Some Special Artex Spaces Over Bi-monoids", "Mathematical Theory and Modeling", An USA Journal of "International Institute for Science, Technology and Education", Vol.2, No.7, 2012, pages 62 - 73.

[5] K.Muthukumaran and M.Kamaraj, "Boolean Artex Spaces Over Bi-monoids", "Mathematical Theory and Modeling", An USA Journal of "International Institute for Science, Technology and Education”, Vol.2, No.7, 2012, pages 74 - 85.

[6] J.P.Tremblay and R.Manohar, Discrete Mathematical Structures with Applications to Computer Science, Tata McGraw-Hill Publishing Company Limited, New Delhi, 1997.

[7] John T.Moore, The University of Florida /The University of Western Ontario, Elements of Abstract Algebra, Second Edition, The Macmillan Company, Collier-Macmillan Limited, London,1967.

[8] Garrett Birkhoff \& Thomas C.Bartee, Modern Applied Algebra, CBS Publishers \& Distributors, 1987.

[9] J.Eldon Whitesitt, Boolean Algebra And Its Applications, Addison-Wesley Publishing Company, Inc.,U.S.A., 1961 